\title{
«Es braucht eine Sensibilisierung der Patienten»
}

\author{
Interview mit Stefan Neuner-Jehlea ${ }^{a}$ Barbara Weil ${ }^{b}$, Linda Hadorn ${ }^{c}$ \\ ${ }^{a}$ Dr. med., MPH, Facharzt für Allgemeine Innere Medizin; ${ }^{b}$ Leiterin Abteilung Public Health, FMH; ${ }^{c}$ wissenschaftliche Mitarbeiterin Abteilung Public Health, FMH
}

\section{Sind Sie direkt mit dem Problem der zunehmend antibiotikaresistenten Bakterien konfrontiert?}

Hin und wieder sehe ich resistente Harnwegsinfektionen oder Wundinfekte. In meinem klinischen Alltag bin ich jedoch selten mit resistenten bakteriellen Infektionen konfrontiert. Die meisten Infektionen unserer ambulanten Patienten können mit einer Erstlinientherapie

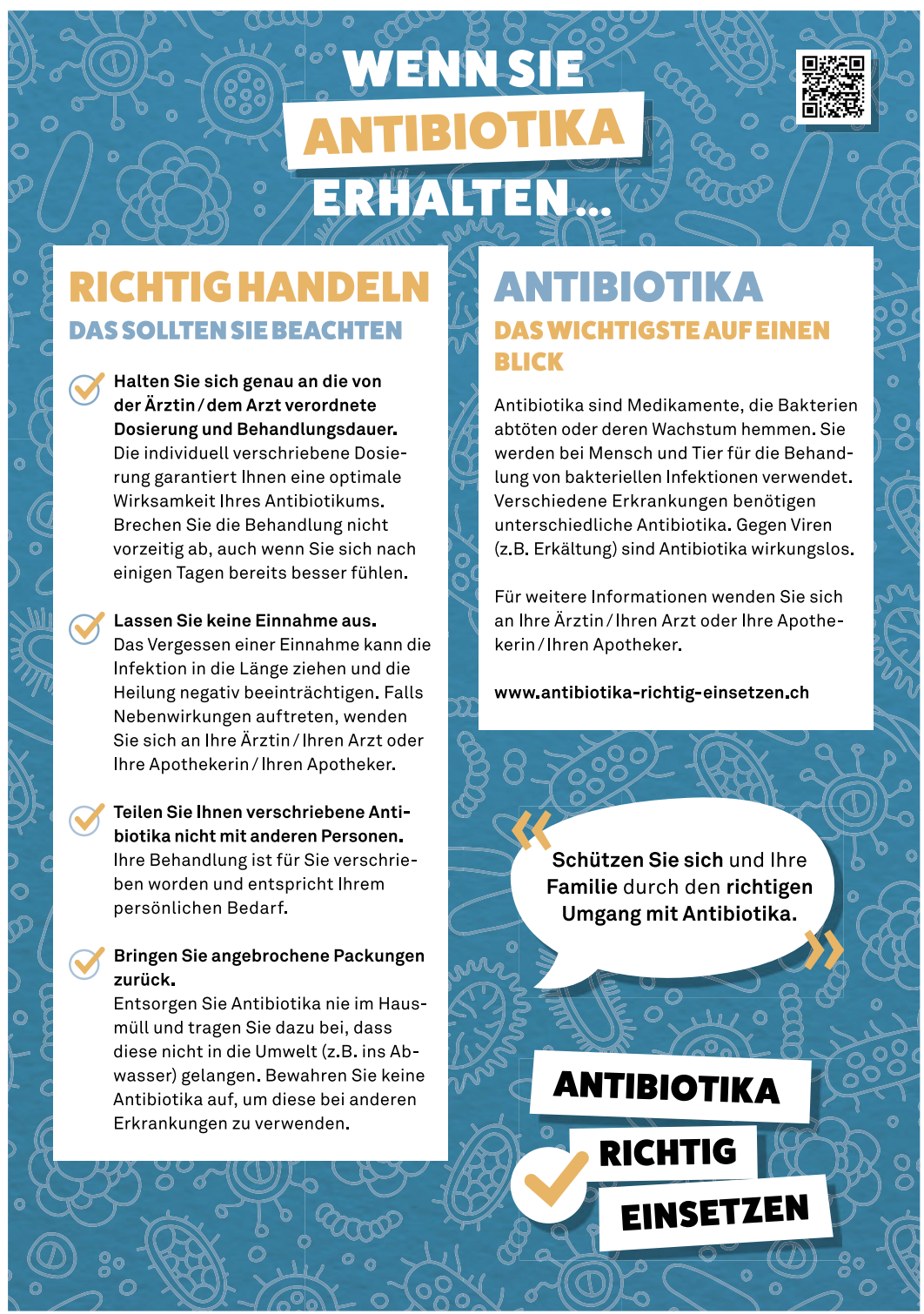

beherrscht werden. Die Einzelfallbeobachtung in der Praxis ist aber naturgemäss nicht aussagekräftig genug. Ich bin mir sehr bewusst, dass Resistenzen in der erweiterten Versorgung, zum Beispiel bei schwierigen oder rezidivierenden Verläufen und im stationären Bereich, ein Problem sind. Hausärztinnen und Hausärzte sind deshalb bemüht, Antibiotika indikationsgerecht einzusetzen, das heisst möglichst nur bei einer hohen Wahrscheinlichkeit für eine bakterielle Ursache und mit Zurückhaltung bezüglich Breitbandantibiotika.

\section{Welches sind für Sie die grössten Herausforderungen} bei der Abgabe bzw. Nicht-Abgabe von Antibiotika? Eine Herausforderung ist die nur lückenhafte Kenntnis von früheren Unverträglichkeiten oder Allergien auf Antibiotika des Patienten. Beim begründeten Abraten von einem Antibiotikum ist die Herausforderung, dies mit der Erwartungshaltung des Patienten in Einklang zu bringen. Das ist zwar in meiner Praxis eher die Ausnahme als die Regel. Hier braucht es eine Diskussion zu Nutzen und Schaden sowie eine Sensibilisierung der Patienten mittels verständlicher Informationen. Ein Kompromiss bei Grenzfällen kann sein, dem Patienten für den Fall einer fehlenden Besserung oder einer Verschlechterung innert definierter Frist ein Antibiotikum (oder ein Rezept dafür) mitzugeben, das er im Falle des Nichtgebrauchs zurückbringen kann. Ein sol-

Ende 2015 verabschiedete der Bundesrat die Nationale Strategie Antibiotikaresistenzen (StAR), um national koordiniert und themenübergreifend gegen die zunehmende Verbreitung von antibiotikaresistenten Bakterien vorzugehen. Als Teil der Umsetzung dieser Strategie haben die Verbindung der Schweizer Ärztinnen und Ärzte FMH, der Schweizerische Apothekerverband pharmaSuisse und das Bundesamt für Gesundheit BAG gemeinsam Materialien entwickelt. Es handelt sich dabei um ein Poster für den Warteberich in der Praxis, einen Erklärfilm zur Verbreitung über soziale Medien sowie ein Faktenblatt zum Umgang mit Antibiotika. Das praktische Faktenblatt im Format A5 kann Patientinnen und Patienten mitgegeben werden. Alle Materialien enthalten sowohl Informationen und Empfehlungen rund um die Antibiotikaeinnahme als auch Gründe, warum bei gewissen Infektionen kein Antibiotikum notwendig ist. Bestellen können Sie diese kostenlos auf der Website: www.antibiotika-richtig-einsetzen.ch 
ches Vorgehen dient als eine Art Rückversicherung. Und die Erfahrung zeigt: Die meisten bringen es unbenutzt wieder zurück.

Im November 2017 lancierte die FMH in Zusammenarbeit mit pharmasuisse und dem BAG ein Faktenblatt zu Antibiotikaresistenzen, welches den Patientinnen und Patienten abgegeben werden kann. Haben Sie dieses bereits verwendet?

Sie liegen in meinem Sprechzimmer bereit und erinnern mich immer daran, dass ich das Thema «angemessener Antibiotika-Einsatz» mit meinen Patienten

\section{Alle können einen Beitrag leisten, damit sich das Problem der Antibiotikaresistenzen nicht ausweitet.}

anspreche. Die wichtigsten Inhalte bespreche ich sowieso mit den behandelten (oder nicht mit Antibiotika zu behandelnden) Patienten, um mein Vorgehen zu erklären und mich ihrer Kooperation zu versichern. Insofern führen die Faktenblätter nicht zu zeitkonsu-

\section{Positiv finde ich vor allem die Erklärungen beim begründeten Verzicht auf ein Anti- biotikum.}

mierendem zusätzlichem Gesprächsbedarf. Ich gebe sie vor allem dann ab, wenn die Patienten noch mehr dazu wissen wollen. Übrigens mag ich solche Diskussionen mit den Patienten gern: Ich versuche wahrzunehmen, wie sie "ticken", und sehe es als Herausforderung für mich, einen guten Draht zu ihnen zu finden. Schliesslich wollen wir zu einer Entscheidung finden, die für beide stimmt.

\section{Gibt es Reaktionen oder Rückfragen auf das Faktenblatt?}

Die Reihenfolge ist bei mir meistens umgekehrt: im Gespräch verweise ich für vertiefte Informationen auf das Blatt. Nach der Lektüre stellen Patienten selten eine Rückfrage.

\section{Wie schätzen Sie den Wissensstand der Bevölkerung im Zusammenhang mit antibiotikaresistenten Bakterien ein?}

Viele Personen haben schon davon gehört, kennen aber die Einzelheiten nicht und wissen auch nicht, wie sie selbst durch ihr Verhalten einen Beitrag leisten kön nen, damit sich das Problem nicht ausweitet. Hier gilt es, die Gesundheitskompetenz zu stärken. Insofern leistet das Faktenblatt einen nützlichen Beitrag, in der Bevölkerung über die Zusammenhänge zu informieren.

\section{Was gefällt Ihnen am Faktenblatt gut und wo, finden} Sie, liegt Verbesserungspotential?

Positiv finde ich die kurz gehaltenen, prägnanten Botschaften und vor allem die Erklärungen beim begründeten Verzicht auf ein Antibiotikum. Das Verzichten ist psychologisch schwieriger für Patienten und benötigt eine besonders sorgfältige Kommunikation. Mittels Erklärfilm oder Plakat im Wartezimmer könnten die Patienten schon vor der Konsultation für das Thema sensibilisiert werden, und zwar für beide Fälle: die Ein nahme wie auch den Verzicht auf ein Antibiotikum

Bildnachweis

Bundesamt für Gesundheit BAG / diff. 